\title{
Dokumentation
}

\author{
Johannes Feest \\ Über den Umgang der Justiz mit Kritik
}

Am Beispiel von juristischen Ratgebern für Gefangene ${ }^{1}$

Das Strafvollzugsarchiv ${ }^{2}$ an der Universität Bremen erhält von Gefangenen immer wieder Briefe wie den folgenden:

»Auf Ihr Schreiben teile ich mit, daß ich in bezug auf Einkauf, Eigengeld, ärztliche Versorgung, Verlegung, Abweichen vom Vollstreckungsplan, Entlassungsvorbereıtung, Briefzensur und Vollzugslockerungen Unterlagen gerne hätte. Auch ersuche ich bei Zusendung um Aufstellung dieser, um den Erhalt kontrollieren zu können« (Bernau, 24. 7.89).

Und manchmal geht die Korrespondenz so weiter:

"Die Post vom 12.7./26. 7.89 von Ihnen an mıch bestätige ich hiermit. Das PS, Zusendung mit gleicher Post, in Ihrem Schreiben vom 26.7.89 kann ich zu meınem Bedauern nıcht bestätıgen. Statt dessen wurde mir hier eröffnet, daß es sich um eine unerlaubte Zusendung handelte, die zu meiner Habe genommen wurde" (Bernau, 9. 8. 89).

"Erneuter Antrag auf Herausgabe mit rechtsmittelfähiger Vorbescheidung wurde erneut abgelehnt mit der Begründung, "Sicherheit und Ordnung der Anstalt" seıen gefährdet beı Aushändigung. Auch stelle man Thre Merkblätter mit dem »Ratgeber für Gefangene" gleich, der durch OLG-Entscheid nicht ausgehändigt werden darf« (Bernau 3.9.89).

\section{Zwei Ratgeber}

Im Jahre 1980 wurden in der Bundesrepublik zwei Bücher publiziert, die den Anspruch erheben, dem Gefangenen brauchbare Informationen zu liefern. So heißt es in der I. Auflage des AK StVollzG:

"Ziel dieses Kommentars ist eine Erläuterung des Strafvollzugsgesetzes für alle an einer gründlichen Reform des Strafvollzuges Interessierten. Sie soll insbesondere dazu dienen, die unmittelbar Betroffenen über Handlungsmöglichketten und deren gegenwärtige Grenzen zu informieren «.

Die anonymen Autoren des anderen Buches bezeichnen es als den

„Versuch, die Grenzen einer bloß beschreibenden Darstellung der Situation der Gefangenen zu überschreiten, indem hier nicht nur Erkenntnisse über diese Institution verbreitet werden sollen, sondern vor allem unmittelbar verwertbare, brauchbare Informationen für diejenigen, die ihr unterworfen sind.*

\footnotetext{
1 Hier sınd insbesondere zwes Bücher gemeint: Kommentar zum Strafvollzugsgesetz, Reihe Alternatıvkommentare, 3. Auflage, Neuwied 1990 (kurz: AK); Ratgeber für Gefangene mıt medizınıschen und jursstischen Hinweisen, 4. Auflage, Berlin 1989. (kurz: , Ratgebers). - Der folgende Text wurde ursprünglich als Vortrag verfaßt und im Rahmen einer Veranstaltung zum so. Geburtstag von Rolf Schwendter vorgetragen. Für die Veröffentlichung wurde er gerıngfügig überarbeıtet und durch Fußnoten ergänzt.

2 Unter diesem Namen exıstıert seıt 1983 an der Unıversıtät Bremen eine Einrıchtung, die es sıch zur Aufgabe gemacht hat, jurıstisch relevante Informationen zum Strafvollzug (Gerichtsentschesdungen, Verwaltungsvorschriften etc.) zu sammeln und an Interessenten weiterzugeben. Viele Gefangenenzestungen drucken die vom Strafvollzugsarchiv verschıckten Infos ab. Und dies führt immer wıeder zu Korrespondenz mit einzelnen Gefangenen.
} 
Der genaue Leser kann schon in der Wortwahl - hier »Betroffene«, dort "Unterworfene« - einen Unterschied zwischen einer eher sozialpädagogisch-reformerischen zu einer mehr sozialrevolutionären Selbstdarstellung der Autoren herauslesen. Beiden Büchern ist jedoch gemeinsam, daß sie ausdrücklich das Gefängnissystem in Frage stellen, also im weitesten Sinne abolitionistisch orientiert sind. Beim Alternativkommentar klingt das so:

„Es ist davon auszugehen, daß der Strafvollzug auch in seıner denkbar aufgeklärtesten Form, die nırgends realisiert ist, mıt eıner Reihe von unauflösbarer Widersprüche und Dilemmas behaftet 1st, z. B.: die Isolierung einzelner 'Schuldiger' reißt diese zugleıch aus dem sozıalen Kontext, auf den eingewirkt werden müßte; Freıwilligkeit des sozıalen Lernens ist unter den Bedingungen der Unfreiheit kaum durchzuhalten oder auch nur festzustellen; Gleıchzeıtıgkeit von Hilfe und Kontrolle führt notwendig zu Rollenkonflikten beım Anstaltspersonal. Der Strafvollzug muß daher soweit wie gesellschaftlich möglich und erträglich abgebaut werden.«

Weniger akademisch-verhüllend - heißt es schon im Vorwort des Ratgeber:

„Das Buch ist vor allem auch für diejenigen wichtig, die, ohne sog. Politısche zu sein, das Strafgesetzbuch verachten und mißachten. Wir sehen es dabei nucht allein als ein technisches Handbuch, sondern auch als einen Beitrag zur inhaltlichen Auseinandersetzung mit dem Knast. Ein Betrag, der die Gefangenen nicht als bloße Opfer der Unterdrückung darstellt, sondern der von den Tatsachen und Möglichkeiten des täglichen Überlebenskampfes - ohne den Preis der Selbstaufgabe - ausgeht... Eine allgemeine Konzeption zur politıschen Organ1sierung des Widerstands im Knast wird man in diesem Buch nicht finden - obwohl die politıschen Meinungen der einzelnen Verfasser nicht ausgeblendet sind.

Beide Bücher haben noch etwas gemeinsam: Sie werden von vielen Gefangenen vergeblich begehrt. Denn den Alternativkommentar können sie sich nicht leisten (er kostet derzeit I88,-DM, weit mehr als ein Gefangener pro Monat zur Verfügung hat). Und der »Ratgeber für Gefangene mit medizinischen und juristischen Hinweisen (der ursprünglich für Gefangene gratis abgegeben wurde, jetzt gegen eine Schutzgebühr von DM $5,-)^{3}$ ist verboten.

\section{Materialien zum Verbot des "Ratgeber für Gefangene mit medizinischen und juristischen Hinweisen"}

Die Verbotsgeschichte des , Ratgeber fängt an im Jahre 198I durch einen Beschluß des Landgerichts Memmingen. Acht Jahre später - angesichts des Memminger Schau-Prozesses gegen einen abtreibungsbereiten Arzt - könnte dies als Ausdruck einer besonders konservativen lokalen Strafjustiz eingeschätzt werden. Aber seither gibt es Verbotsentscheidungen der Gerichte quer durch die Bundesrepublik. Ich zähle hier nur die mir bekannten Stationen auf: Hamburg, München, Saarbrücken, Gießen, Frankfurt, Zweibrücken, Mainz, Frankenthal, Kleve, Ulm, Kassel, Limburg, Krefeld, Düsseldorf, Braunschweig, Freudenstadt, Regensburg, Berlin, Trier, Nürnberg, Arnsberg, Hamm, Darmstadt, Stuttgart und zuletzt nochmals Frankfurt im Jahre 1989. Diese Entscheidungen betreffen sowohl Strafgefangene wie auch Untersuchungsgefangene.

\section{Warum Gefangene den, Ratgeber nicht lesen dürfen}

Hauptargument in den Gerichtsentscheidungen ist die Behauptung, der $>$ Ratgeber würde bei den Gefangenen »aggressives Verhalten erzeugen oder verstärken «4; er sei

\footnotetext{
3 Der Ratgeber (4. Auflage 1989) Ist vergriffen. Eine Neuauflage wird vorberestet durch den *Vereın zur

Förderung von Kultur und Information für Gefangene e.V.", Gnessenaustraße 2a, roco Berlin 6r.

4 OLG Hamburg, 7. 5. $198 \mathrm{I}-\mathrm{I}_{\mathrm{W}} \mathrm{W}_{144} / 8 \mathrm{I}$ (U-Haft).
} 
"geeignet, bei Gefangenen eine haßvolle und aggressive Oppositionshaltung gegenüber dem Vollzug und den Bediensteten der Anstalt hervorzurufen «s.

"Kämen diese Textstellen in die Hand des Untersuchungsgefangenen, müßte besorgt werden, $\mathrm{da} ß$ er und andere Gefangene, denen sie zur Kenntnis gelangen, zu aggressivem und ordnungswidrıgem Verhalten gegenüber den Anstaltsbediensteten veranlaßt würden « ${ }^{6}$.

Und noch etwas umständlicher und umfassender das Landgericht Regensburg7, wonach das Buch geeignet sei,

»enne haßvolle und aggressive Oppositıonshaltung vor allem gegen die Bediensteten der JVA, aber auch gegen Polizeıorgane und Justiz zu begründen. Daraus ergibt sich die nıcht auszuschließende Gefahr, daß andere Gefangene, die Zugang zu dem Druckwerk erhalten, den im Buch unrichtig wiedergegebenen Angaben Glauben schenken und sıch zu aggressivem Verhalten verführen lassen. $\varkappa^{8}$

Nun behaupten selbst die größten Kritiker des >Ratgeber` nicht, daß er rundherum schlecht sei. Vielmehr hat schon 198 I das OLG Hamburg eingeräumt, daß der Ratgeber auch sachliche Informationen und einzelne Darlegungen enthält, "gegen die, für sich betrachtet, keine Einwendungen zu erheben sein würden «" Und das OLG Frankfurt hat erst kürzlich dem >Ratgeber< bescheinigt, er enthalte »auch unverfängliche und nicht zu beanstandende Passagen ${ }^{10}$. Vereinzelte Untergerichte haben sogar vorgeschlagen, nur die beanstandeten Passagen zurückzuhalten und die übrigen den Gefangenen auszuhändigen ${ }^{1}$. Dies würde sicherlich dem Verhältnismäßigkeitsgrundsatz entsprochen haben, der zu den zentralen Prinzipien unseres Verwaltungs- und Verfassungsrechts gehört. Aber die Obergerichte haben dem bisher energisch widersprochen, wobei das Bild des »roten Fadens« zentraler Bestandteil der Begründungen ist: Schon das OLG Hamburg hat 198 I von der »wie ein roter Faden das ganze Druckwerk durchziehenden Tendenz « gesprochen. Und nach Meinung des OLG Frankfurt' ${ }^{2}$ ist "auf die das gesamte Druckwerk beherrschende und es wie ein roter Faden durchziehende Tendenz abzustellen, durch die bei dem Gefangenen ein aggressives Verhalten erzeugt und verstärkt werden soll «. Außerdem sei es den Vollzugsbehörden nicht zuzumuten, das Druckerzeugnis "Seite für Seite auf die zu beanstandenden Stellen durchzulesen «'3 oder "durchzuforsten ${ }^{14}$. Und das OLG Frankfurt hat noch neuerdings auf die Gefahr hingewiesen, »daß sich auch in auf den ersten Blick unverfänglichen Kapiteln nicht mehr hinnehmbare Passagen befinden «'s. Und damit war und ist der > Ratgeber < insgesamt geächtet und von der Aushändigung an Gefangene ausgeschlossen.

s OLG Hamm, 9. 2. 1988 - I Vollz (Ws) $414 / 87$.

6 OLG Frankfurt, 22.6.1989-3 WS 21 s/89 (U-Haft).

7 LG Regensburg, 23.1. 1986 - StVK 197/83 (2b).

8 Das Grundmuster dieser Argumentation findet sich bereits in einer älteren Entscheıdung des OLG Nürnberg (9. 5. 1974 - VAs 2/74, abgedruckt 1n: Vollzugsdienst 3/1975, S. 1 1 ), wo es um die Herausgabe von zweı Büchern an eınen Gefangenen gıng: „Eine Durchsıcht des Taschenbuches Justız - die stille Gewalt< ergibr, daß sıch eınzelne Abhandlungen ım wesentlichen darauf beschränken, möglicherweıse tatsächlich bestehende Mängel der Justız und deren Vertreter zu kritısıeren. Dabeı wird auch von Klassenjustız gesprochen. Diese eınseitıge Darstellung und häufig abwertende Betrachtungsweıse erschweren den Strafgefangenen die Einsıcht in ihr eigenes - ehedem unrechtes - Verhalten und ihre Wiederengliederung in die Gesellschaft. Sie begünstıgen auch eınen Autorı̈ätsabbau ım Strafvollzug selbst... Das gleıche gilt für den Inhalt der Druckschrift "Ich lerne Karate«.

9 OLG Hamburg, 7.5. 1981 - I WS I 44/81.

10 OLG Frankfurt, 22.6.1989-3W/s 215/89.

11 Z.B. LG Arnsberg, 13.11.1987-1 Vollz 411/87.

12 OLG Frankfurt, 10.11. $1982-3 \mathrm{Ws} 783 / 82$.

13 OLG Frankfurt, 10.11, $1982-3$ Ws $783 / 82$.

14 Wie die für die JVA Straubıng zuständige Strafvollstreckungskammer (LG Regensburg, 23. 1. 1986 StVK $197 / 83$ (2b)) etwas deftiger formuliert.

is OLG Frankfurt, 22.6. $1989-3$ WS $215 / 89$ 
Auf die juristische Seite der Sache soll hier nicht näher eingegangen werden. Eine juristische Gegenargumentation ist an anderer Stelle versucht worden ${ }^{16}$. Auf eine Schwierigkeit sei jedoch hingewiesen: Die Logik juristischer Argumentation könnte dazu verführen zu behaupten, von den Texten des 'Ratgeber gehe überhaupt keine Gefahr für die Anstaltssicherheit, die Anstaltsordnung oder das Ziel des Vollzuges aus. Und das ließe sich auch durch den Hinweis belegen, daß es noch heute mindestens eine Anstalt gibt (die JVA Bremen-Oslebshausen), die den >Ratgeber nach wie vor an Gefangene aushändigt. Aber gesetzt den Fall: der >Ratgeber ‘ würde doch aggressives und ordnungswidriges Verhalten »erzeugen " oder zumindest verstärken? Was, wenn dann ein Gefangener schon vor seiner Inhaftierung und während eines Hafturlaubs in dem Buch gelesen (oder Karate gelernt) hat? Nach der Logik der Gerichtsentscheidungen müßte ein solcher Gefangener als nicht vollzugstauglich von Gefängnissen ferngehalten werden.

\section{Was Gefangene nucht lesen dürfen}

Es ist nicht ganz einfach herauszufinden, was Gefangene nicht lesen dürfen bzw. was die Gerichte für »nicht mehr hinnehmbare Passagen « halten. Denn, wie schon das OLG Hamburg ${ }^{17}$ sagt: "Der Zweck dieser Maßnahme schließt es aus, die zu beanstandenden Darstellungen, Formulierungen und Ausführungen hier im einzelnen wiederzugeben. « Glücklicherweise sind nicht alle Gerichte so streng gewesen, haben entweder die beanstandeten Seitenzahlen oder sogar kleine Beispielszitate angegeben. Im folgenden werden vier der am häufigsten beanstandeten Passagen wiedergegeben.

\section{Die polizeiliche Vernebmung}

»Nach der Festnahme findet nun das polizeiliche Verhör auf dem Revıer statt. Dabeı mußt Du Dir unbedingt klar darüber seın: entweder man sagt gar nıchts, was generell am besten ist, oder man nennt sein Alibi, wenn man ein sicheres hat und mit der eigenen Aussage niemand anderen in Gefahr bringt (ist vor allem wichtig, wenn Du mit mehreren zusammen einfährst). Dies kannst du jedoch nie richtig überblicken.

Deshalb immer erstmal: keine Aussagen. Wenn Du Glück hast, erwischt Du einen Beamten, der nicht sonderlich unglücklich darüber 1st, weil Du ihm damit die Arbeit ersparst, ein Vernehmungsprotokoll zu schreiben. Kurz nach der Festnahme bist Du natürlich erstmal. aufgeregt, sauer und vielleicht auch niedergeschlagen und erschöpft. Diesen Zustand versuchen die Vernehmungsbeamten erfahrungsgemäß für sich auszunützen, d.h. sie setzen Dich mal unter Streß, mal behandeln sie Dich richtig freundlich mit Kaffee und Zigaretten und so, um Dich sweichzukochen`. Diese Art von nervlichen Wechselbädern soll Dich mürbe und müde machen, damit Du möglichst schnell möglichst viel aussagst. Somit haben sie nicht allzu viel Arbeit mit Dir (auch für Polizisten ein wichtiger Aspekt, denn auch die sind faul), und andererseits können sie Erfolge vorweisen, was wiederum gut für ihre eıgene Psyche und Beförderung 1st. « ${ }^{18}$

Deskriptiv ist diese Passage schwerlich zu beanstanden, und die daraus folgende Handlungsanweisung »keine Aussagen« wird jeder Strafverteidiger, der sein Geld wert ist, dem Mandanten geben, der ihn aus dem Polizeigewahrsam anruft.

\section{Die nuchtoffizıelle Kommunikatıon}

"Dazu gehört das Sprechen am Fenster. Schon am ersten Tag im Knast wird man merken, daß der Knast sich mıt sich selbst unterhält. Man sprıcht von Zellenfenster zu Zellenfenster... Das

I6 Johannes Feest und Wolfgang Lesung, Anmerkung zu LG Hamburg, NStZ 1988, S. 333 ff. Vgl. auch Claus Bertram/Konrad Huchtıng, $\$ 73$ Rz. 6, AK StVollzG, 3. Aufl., Neuwied r99o.

17 OLG Hamburg, 7.5. 198 I - I WS I 44/8I.

18 ,Ratgeber<, Kap. 1.2; S. 3. 
Pendeln ist ebenso in allen Knästen üblich, wird allerdings von den Grünen ımmer zu verhindern versucht. Unter Pendeln versteht man das gegensertige Zuwerfen eınes Gegenstandes, der an einer Schnur (Pendelschnur) notfalls noch mit einem Gewicht daran, befestıgt ist. Gependelt wird von Fenster zu Fenster. Es ist schwierı, wenn die Fenster mit einem Maschendraht verbaut sind oder wenn Sichtblenden davor sind. Aber auch da finden immer welche eine Möglichkeit...

Man muß natürlich damit rechnen, besonders bei Sachen, die sich am Fenster abspielen, daß man von dem Wachhabenden im Hof gemeldet wird, der mit einem Fernglas die Fenster absucht. Ein welteres übliches Mittel, mit anderen in Verbindung zu kommen - wenn ihre Zelle zugeschlossen ist oder z. B. wenn sie isoliert sınd oder auf einer anderen Station liegen ist ein Kassiber, en Stück beschriebenes Papier, das meistens winzig klein ist, um es notfalls aufessen zu können, und das man entweder selbst durch eine Tür schiebt oder von einem Hausarbetter bzw. einem anderen Gefangenen überbringen läßt... Nach der Hausordnung ist diese Art der Kommunikation verboten und kann mit Hausstrafen belegt werden. Aber die Hausordnung ist in vielen Punkten nur dazu da, zu zeigen: Wir können Euch alles verbieten, wenn wir wollen. Wenn diese Hausordnungen strikt von den Grünen eingehalten würden, wäre u. U. auch das Wertergeben von Zeitungen und das aus dem Fenster Sehen, ja oft selbst das Sprechen verboten. Trotzdem wird beim Hofgang gesprochen und aus gerade den verbotenen Fenstern hinausgeguckt. Die Grünen versuchen ihr Bestes, das alles zu verhindern. Aber das liegt an den Vorschriften: Wenn sie übertrieben sind, dann sind sie eben nur durch übertriebene Anstrengungen einzuhalten, und die Beamten scheuen die übertriebene Anstrengung «" (Kapitel 3.3).

Die beschriebenen Formen nichtoffizieller Kommunikation sind innerhalb und außerhalb der Gefängnisse derartig wohlbekannt, daß sie kaum Informationswert haben.

\section{Die Begegnung mit dem Psychologen}

"Wir gehen hier - wie schon im bisher Gesagten - von dem Psychologentyp aus, mit dem man schlimmstenfalls rechnen muß. Dabei ist uns klar, daß es auch andere gibt. Es ist auch nicht die Regel, daß Dich der Psychologe ständig bedrängt. Im Gegenteil. In der Regel wirst Du ihn kaum zu Gesicht bekommen.

Eine mögliche Verhaltensstrategie gegenüber dem Psychologen ist vielleicht, sich alles erklären zu lassen und z. B. vom Psychologen zu fordern, daß er Dir den Psycho-Test erklärt. Er wird es nicht tun, und er wird es auch nicht können. Aber es ist eine Möglichkeit, ihm seine Autorität wegzunehmen. Vielleicht wird er versuchen, auch dieses Verhalten von Dir zu vermerken, indem er Dein Mißtrauen als Symptom einordnet. Aber dem kann man vorbeugen, indem man gleich im nächsten Atemzug fragt, ob er eigentlich vorhat, diese Frage als Symptom zu bewerten. Dazu gehört eine gewisse Schlagfertigkeit und Sachkenntnis, die allerdings die meisten nicht haben werden.

Man müßte also Methoden finden, die jeder anwenden kann, auch ohne spezıelle Kenntnisse in der Psychologie. Die allgemeinste und einfachste Methode ıst die Verweigerung. Man kann eine Erklärung abfassen, in der steht, daß man diese Testmethoden als einen Eingriff in seine Persönlichkeitsrechte betrachtet, die nach dem Grundgesetz geschützt sind, und daß man deswegen überhaupt jede Beteiligung daran verweigert. Die Erklärung könnte etwa so aussehen: IIch lehne eine Mitwirkung an einer psychologischen Untersuchung ab, weil ich die dabei angewendeten Methoden als einen Eingriff in meine Persönlichkeitsrechte betrachte, da ich weder erkennen kann, was sie beinhalten, noch wozu ihre Ergebnisse verwendet werden, gez.<

Den Zettel unterschreibt man und gibt ihn dem Psychologen. Soll er ihn dann in seine Mappe heften. Auf weiteres Fragen von ihm, warum man die Mitwirkung verweıgert, darf man dann allerdings nicht mehr antworten. Wenn ihm klar ist, daß man nicht mehr mit ihm reden will, wird er es nicht mit Gewalt versuchen. Reden ist nicht zu erzwingen. Damit ist seine Tätigkeit an Dir praktisch ausgeschaltet. Er kann höchstens noch ein graphologisches Gutachten von Deiner Schrift anfertigen oder anfertigen lassen. Dagegen wehrst Du Dich, indem Du alles mit Maschine schreibst oder in Blockbuchstaben. ${ }^{20}$

19 Ratgeber, Kapitel 3.3, S. 9 f.

20 Ratgeber, Kapitel 5.7, S. $30 \mathrm{f}$. 
Diese, gewiß provokante Passage kann sich juristisch darauf stützen, daß das Strafvollzugsgesetz die Gefangenen nicht zur Mitwirkung an ihrer Behandlung verpflichtet. ${ }^{21}$

\section{Wie man im Knast gesund bleiben kann}

Mit diesen Kapiteln beginnt ein umfangreicher Abschnitt über "Krankheit - medizinische Versorgung und Selbsthilfe«. Darin sind sowohl Anleitungen zur Krankheitsprävention (Gymnastik, Atemübungen, Autogenes Training, Massagen etc.) als auch von spezifischen Knast-Krankheiten und ihren Ursachen (*krankmachende Haltung« etc.), häufigen Gesundheitsbeschwerden, Notfällen und speziell von Frauenkrankheiten die Rede. Beanstandet wird von der Rechtsprechung vor allem die erste Seite des gesamten Abschnitts:

"Auch Im reformierten Strafvolizug soll der Gefangene mit Zuckerbrot und Pettsche, mit Verhaltenstraining und guten Worten zerbrochen werden: Wer sıch nicht fügt, wird kleingemacht. Auch wenn viele das nicht wahrhaben wollen und niemand gerne von seinen Niederlagen erzählt: Isolatıonshaft, Beruhıgungszellen, Greif- und Schlägerkommandos, die geduldete, manchmal auch geförderte Brutalität gegenüber schwächeren Mitgefangenen, Dauerbeleuchtung, Trennscheiben, Rede-, Schreib- und Besuchskontrollen sind die Petrschenhiebe, die die Gewährung selbstverständlicher Rechte wıe Sport, Gottesdienst, gemeinschaftlichen Umschluß, Radio, Post und Fernsehen als besondere Hilfe erscheinen lassen sollen. Das alles dient in seıner Vielfalt und Willkür dazu, das Rückgrat des Gefangenen zu brechen, ihm seıne Persönlichkeit zu nehmen.

Der Lebensraum wird auf wenige Quadratmeter eingeengt, Licht und Luft reichen nicht zum Atmen. Bewegung wird auf den Innenhofrundgang beschränkt. Sport bleibt nur als Belohnung erlaubt und dann meist als Konkurrenzkampf der Gefangenen gegeneinander. Gespräche, Gefühle und Gemeınsamkeıten werden als Belohnung organısıert und festgefügten Formen und Hierarchien unterworfen. Und doch hat jeder Gefangene für sıch schon Überlebensstrategien entwıckelt, die ihm helfen, seine Individualität, seine Persönlichkeit zu bewahren. Die Erfahrungen Einzelner wollen wir in den folgenden Abschnitten benutzen, um Ratschläge zum Überleben geben zu können. Dabeı ist es wichtıg, zu erkennen, welche Teile meiner Persönlichkeıt der Strafvollzug ändern bzw. zerstören will, um dagegen Widerstand leisten zu können. Aus den Anregungen zur körperlichen Kräftrgung, zu Entspannungs- und Konzentrationsübungen, zur Ernährung und zur Selbstbehandlung kann sich jeder das aussuchen, was ihm im Moment wichtigste Waffe ım Überlebenskampf zu sein scheint und was er meint, am ehesten lernen zu können. Und vor allem sollte er sich sagen, daß jeder kleine Schritt schon wichtig genug ist und nicht ernst genug genommen werden kann. Seht Euch das Verhalten und Vorgehen der Vollzugsbeamten an, denen ist kein Schritt zu kleın, um den Gefangenen kaputt zu machen. $\otimes^{22}$

Sprachlich erinnert diese Passage an Texte von Gefangenen der RAF und ihres Umfeldes. Inhaltlich erscheint mir die Charakterisierung der Vollzugsbeamten völlig überzogen (denn auch dort gibt es viele verschiedene Verhaltensstile, wie dies auch an anderen Stellen des , Ratgeber zutreffend betont wird). Dafür ist der ,Ratgeber schon frühzeitig, wenn auch bisher ohne Erfolg kritisiert worden. ${ }^{23}$

2I Johannes Feest, $\$_{4}$ Rz. 5 , AK StVollzG, 3. Aufl., Neuwied 1990 m.w.N.

22 ,Ratgeber, Kapicel 13.1, S. 1.

23 *Widerspruch anmelden müssen wir jedoch beı der Unterstellung, die sıch - teils offen, teils zwıschen den Zeilen - Immer wieder aufdrängt, daß die Bemühungen progressiver Mitarbeiter alleın der Zerstörung der Persönlichkeıt mıt anderen Mitteln dienen ... Trotz der Krıtik an dem Ratgeber erscheınt uns der Nutzen für die Betroffenen größer als die Mängel aus der Sicht der Außenstehenden. Es wäre zu wünschen, daß in späteren Auflagen die Diskrımınıerung des Anstaltspersonals aufgegeben wird zugunsten der Krıtik an den Instıtutıonen. Es steht nämlich zu befürchten, daß Anstaltsleıter, Personal und Behörden aus Mangel an Souveränıtät die Benutzung verhındern werden und der Ratgeber die Betroffenen selber nıcht erreıcht« (Edelgart Quensel/Erich Joester, StV 1981, 212). 


\section{Erste Metamorphose des, Ratgeber}

Mitte 1984 baten die Herausgeber der 2. Auflage des 'Ratgeber`, zu denen keine Juristen mehr gehörten, das Strafvollzugsarchiv der Universität Bremen um Hilfe bei der Überarbeitung des juristischen Teils. Zunächst beschränkte sich die Kooperation darauf, daß wir (meine Mitarbeiter und ich) den $>$ Ratgeber-Leuten neuere Gerichtsentscheidungen zugänglich machten. Aber bald stellte sich heraus, daß auch die 2. Auflage des $>$ Ratgeber das Verbotsschicksal der ersten teilte. Und dies brachte uns auf die Idee, den juristischen Rat gewissermaßen häppchenweise zu verschicken. Wir begannen also in Bremen damit, auf der Basis des $>$ Ratgeber - Textes Merkblätter mit "Musterbegründungen« zu einzelnen Problembereichen herzustellen. Mit der Zeit entstanden auf diese Weise is juristische Merkblätter, deren Inhalte von der Arbeitspflicht bis zur Zweidrittel-Entlassung reichten. Die Intention war dabei eine doppelte: Zum einen schien uns die Chance größer, daß Gefangene auf diesem Wege die gewünschte Argumentationshilfe erhielten. Zum anderen wollten wir die Anstalten und Gerichte dazu zwingen, ihre Verbotspolitik zu überprüfen. Letzteres schien deshalb besonders aussichtsreich, weil der juristische Teil des >Ratgeber in den Verbotsentscheidungen niemals ausdrücklich beanstandet worden war. Wir versäumten es nicht, zu Beginn unserer Merkblätter darauf hinzuweisen, daß es sich um - von uns überarbeitete - Teile des , Ratgeber handelte. Und wir verschickten diese Merkblätter an sämtliche Gefangenenzeitungen und an individuelle Gefangene.

Es gab die erwarteten Schwierigkeiten, aber sie hielten sich zunächst in Grenzen. Ich will dies an einigen Beispielen illustrieren:

- Von einem Gefangenen aus Bielefeld hörten wir, ihm sei eine Briefsendung von uns nicht ausgehändigt worden, mit der Begründung, daß der Inhalt der Sendung das Vollzugsziel gefährde. Zudem enthalte die Sendung Auszüge aus dem »Ratgeber für Gefangene " und - wie ihm ein Bediensteter wörtlich sagte: "So ein linker Kram kommt mir nicht ins Haus «. Aufgrund unserer Beschwerde bei der Aufsichtsbehörde wurden die Merkblätter nach zweieinhalb Monaten an den Gefangenen ausgehändigt.

- Eine Gefangenenzeitung durfte nicht erscheinen, weil sie eine Karikatur von Marie Marcks und eines unserer Merkblätter abdrucken wollte. Auf Beschwerde wurde die Verfügung des Anstaltsleiters durch das zuständige Ministerium aufgehoben. Die Musterbegründungen durften abgedruckt werden. Nicht aber die Karikatur, die eine Gruppe von Menschen zeigt, die in einen Brunnen urinieren, während am Rande des Brunnens ein junger Mann eine Flasche Alkohol an den Mund setzt. Begründung: Die Karikatur könnte jugendliche Gefangene zum Alkoholkonsum animieren. Aber das nur nebenbei!

\section{Erste Metastase der Verbotsjustız}

Größere Schwierigkeiten entstanden allerdings, nachdem ein Gefangener, mit dem ich schon länger in Korrespondenz stand, strafweise in eine andere Anstalt verlegt und dort auch gleich noch mit drei Monaten »Beschränkung des Verkehrs mit der Außenwelt « ( $\mathbb{S}$ 103 Abs. I Ziff. 8 StVollzG) belegt wurde. Er konnte mir dies gerade noch mitteilen, bevor er total isoliert wurde. Daraufhin entschloß ich mich, ihm unser neuestes Merkblatt über »Isolierende Maßnahmen« zuzusenden. Es beginnt mit den Worten: 
"Wenn $\mathrm{Du}$ von anderen Gefangenen isoliert worden bist oder werden sollst, dann ist juristische Gegenwehr besonders nötig und auch besonders schwierı. Fordere die sofortıge Benachrichtigung Deines Anwaltes oder eıner anderen Vertrauensperson (wenn Du Ausländer bist: ennes Dolmetschers). Versuche Kontakt mit dem Anstaltsbeirat aufzunehmen (der Dich unüberwacht aufsuchen darf: $\$ 16_{4} \mathrm{Abs} .2 \mathrm{StVoll} z \mathrm{Z}$ ), und stelle umgehend einen Eilantrag bei der Strafvollstreckungskammer.«

Pünktlich drei Monate später erhielt ich Nachricht von dem Gefangenen. Mein Schreiben sei ihm jetzt ausgehändigt worden, allerdings ohne die Beilage. Als Begründung sei folgendes angegeben worden:

"Die Rechtmäßigkeit der Anhaltung des Ratgebers für Gefangene mit medizınischen und jurıstıschen Hinweisen wurde bereits durch die Strafvollstreckungskammern bestätıgt. Eine Aushändigung von Teilen dieses Ratgebers würde der Anhaltung zuwiderlaufen.«

Dagegen rief ich die Strafvollstreckungskammer des Landgerichts Augsburg beim Amtsgericht Nördlingen in Donauwörth an und argumentierte, daß jedenfalls die »Musterbegründungen« bisher noch nie als Grund für das Verbot des , Ratgebers genannt worden waren. Das Gericht erließ darauf folgenden Beschlu $3^{24}$ :

»Die sog. Musterbegründungen für Anträge und Beschwerden, die auf dem ২Ratgeber für Gefangene mit medizınischen und juristischen Hinweisen< beruhen, sind von Mitarbeitern des - Vollzugsarchivs‘, zu denen auch der Antragsteller zählt, erarbeitet.

Ziel und Inhalt der >Musterbegründungen ist es, Strafgefangene zur juristischen Gegenwehr gegen Disziplinar- und Sicherheitsmaßnahmen zu bestimmen. Diese Gegenwehr wird von den Verfassern als snotwendig، erachtet (vgl. I der >Musterbegründungen`), ohne daß sie darauf abstellen, ob die von Seiten der Anstalt getroffene Maßnahme tatsächlich berechtigt ist oder nicht. Sie empfehlen vielmehr folgendes:

-Wenn Du von anderen Gefangenen isoliert worden bist oder werden sollst, dann ist juristısche Gegenwehr besonders nötı und auch besonders schwerıg, ... und stelle umgehend einen Eilantrag bei der Strafvollstreckungskammer. Sie formulieren infolge dann diesen Eilantrag so vor, daß lediglich die angegriffene Maßnahme und die Gründe für die Anfechtung der Maßnahme, die ebenfalls zum Teil vorgegeben sind, in die Rechtsmittelschrift enzusetzen sınd. Die wahlweise angebotenen Begründungsmuster werden unter der Überschrift ,Argumentiere wie folgt< angeboten.

Diese Argumentationsmuster sind einseitig ausgewählt und Jassen bei Gefangenen, die oftmals - falsche Hoffnung entstehen, sie werden mit ihrem solchermaßen erhobenen Rechtsmittel Erfolg haben. Im Falle eines Mißerfolges aber werden sie glauben, Opfer einer unsachgemäßen willkürlichen Entscheidung zu sein, denn aufgrund der >Musterbegründungen sind sie nıcht in der Lage zu erkennen, daß gewichtige Meınungen in Literatur und/oder Rechtsprechung der in den >Musterbegründungen` vertretenen Auffassungen entgegenstehen. Dieses Manko der , Musterbegründungen< wird auch nicht dadurch ausgeglichen, daß darauf hingewiesen wird, die ene oder andere dort vertretene Auffassung sei streitig und auf umfangreiche Kommentarliteratur hingewiesen wird. Diese Tatsache, daß eine Auffassung streitig ist, sagt noch lange nichts darüber aus, welches die überwiegende oder gar herrschende Meinung in Rechtsprechung oder Literatur ist. Diese Tatsache ist für Laien, zu denen in der Regel die Inhaftierten gehören, auch nicht ohne weiteres erkennbar. Sie werden auch im allgemeinen nicht dazu in der Lage sein, die vom Antragsteller oder Verfasser angegebenen Kommentarstellen nachzulesen und so festzustellen, daß die vom Antragsteller vertretene Auffassung ihnen nicht zum Erfolg verhelfen wird.«

Letzteres ist nur allzu wahr, da in den meisten Anstaltsbüchereien die Kommentare zum Strafvollzugsgesetz nicht oder nicht in genügender Anzahl vorhanden sind, ganz zu schweigen von den einschlägigen juristischen Fachzeitschriften. Gegen die Entscheidung der Strafvollstreckungskammer wäre prinzipiell der Rechtsweg zum Oberlandesgericht möglich gewesen. Nach gründlicher Überlegung verzichteten wir jedoch darauf, da wir uns vom OLG Nürnberg keine Abhilfe versprachen ${ }^{25}$.

24 LG Augsburg, 20.5. 1987 - NöStVK 29/87.

25 Eine weitere Metastase zeıgte sıch in diesem Fall eın Jahr später. Aus eınem Brıef des betreffenden Gefangenen: *Hatte letzte Woche ,Anhörungs wegen Führungsaufsıcht. Naja, das Übliche. Die Anstaltsleıtung hatte die gleıche Stellungnahme von der Drittelanhörung nochmal benutzt (als ob dazw1- 
$\mathrm{Zu}$ Weihnachten 1987 hatte die links-alternative >Tageszeitung، (taz) ebenfalls die Idee, den verbotenen 'Ratgeber< unter die "gefangenen Leute« zu bringen. Die Redaktion verschickte ein »subversives Weihnachtsgeschenk « an diejenigen Gefangenen, die ein Gratis-Geschenkabonnement erhielten:

»Lieber Kollege!

du bist in strafhaft und da habe sch all das, was dich aus rechtlicher sicht angeht, aus dem gefangenenratgeber kopiert. nun wirst du fluchen, da die schrift so klein ist und durch die haft deine augen so schlecht sind. glaub mir, es gıng nicht anders, und du sollst diesen rechtlichen teil ja auch nicht in einem zug durchlesen, sondern ihn bunkern, um ihn wieder auszugraben, wenn du ihn in einem punkt als argumentationshilfe gebrauchen kannst... so, lieber kollege, laß den kopf nicht hängen und laß dich nie von der dampfwalze knast unterkriegen. freiheit und glück:

Ralf-Axel Simon."

Diesem Schreiben war als Beilage der gesamte rechtliche Teil des >Ratgeber<, einschließlich sämtlicher Musterbegründungen, in starker Verkleinerung, aber so gerade noch lesbar beigefügt.

Für die Reaktion der Anstalten und der Gerichte nur drei ausgewählte Beispiele:

- D.S. aus der JVA Diez am 5. I. 1988 an die taz:

- "Lieber Axel,

am heutigen Tag gegen $\times 1.30$ Uhr erhıelt 1ch Demen Brief ausgehändigt, um 16.10 Uhr wurde dieser mir wieder weggenommen, mit dem Bemerken, diese Anordnung se1 von vorne gekommen (Verwaltungswasserkopf). Ich bekäme noch Bescheid, weshalb oder ähnlich. Zufällig habe ıch allerdings das Kleıngedruckte gelesen, und was im Kopf ist, wird gespeichert.

Meine Resozialisierung, die mir von setten der Anstalt als Vorbild vorgeführt wird, ist: Verlogensein, Betrügen, Rechtsverdrehung, Feegheit, Schwachsinn, Sich-nicht-erinnern-können, Geheımnıskrämerei u.v.a.m. Hätten die Gefangenen nur ein Drittel der kriminellen Energie aufgewandt, die hier von ziviltragenden Funktıonsträgern täglich praktiziert wird, der Knast wäre nur zu einem Viertel belegt.“

- Verfügung des OLG Stuttgart v. 27. I. 1988

»in der Strafsache (sic!) gegen E.H. und zwe1 andere wegen Mitgliedschaft in einer terroristischen Vereinigung u. a....«:

„Die Postsendung der >Tageszeitung bzw. des Ralf-Axel Simon ... an die Angeklagte H. wird nicht an diese ausgehändigt, sondern zu ihrer Habe genommen.

Gründe:

Der Inhalt der Broschüre ist geergnet, die Ordnung in der Anstalt zu gefährden. Die Schrift enthält neben sachfremde Zwecke verfolgenden, Ratschlägen auch grobe Beleidigungen von Anstalts- und Justizbehörden «.

- Der stellvertretende Leiter der JVA Celle läßt das Schreiben der taz mit folgender Begründung anhalten:

"Das Schreiben der taz ... an den Strafgefangenen D. W. wird angehalten. Es ist unleserlich $(\$ 36$ Abs. ^ Ziff. 6 StVollzG). Lt. Anschreiben handelt es sich um Auszüge aus dem >Gefangenenratgeber،. Dieser ist wegen semer vollzugsfeindlichen Grundtendenz vom Bezug allgemein ausgeschlossen. Die Kontrolle der in Ablichtung übersandten Auszüge hat deshalb ganz besonders sorgfältig zu erfolgen, um so verhindern, daß die zu beanstandenen Teile des

schen nıcht fast zweı Jahre liegen würden und 'ne komplette Lehrausbildung). Am lustigsten war ja, als mır der Richter die ganzen, Vorwürfes nochmal eröffnete. Darunter •Der Gefangene bekennt sıch zu Anarchısmus und Abolitıonısmus c. Dabeı kam er beım Abolitionısmus verbal ıns Stolpern, und prompt fragte er auch gleıch, was das seı. Erklärte ihm dann, daß dies 'ne Richtung der Krımınologıe seı, die auch von renommierten Leuten dieses Wissinschaftszweiges vertreten würde. Er sah mich nur verdutzt an und fuhr dann weiter $1 \mathrm{~m}$ Text. Konnte mır dann nıcht verkneifen nachzuhaken, ob er denn nıchts dabe fände, Führungsaufsıcht u. a. damıt zu begründen, daß ıch mıch zu 'ner krımınologıschen Posıtıon bekenne, die auch von führenden Wissenschaftlern vertreten wird. Bekam aber keıne Antwort darauf.* 
Werkes in Raten in die Anstalt gesandt werden können. Diese Kontrolle kann indes vorliegend niemand zugemutet werden, da es sich um sieben DIN A 4 -Seiten in winzigstem Druck handelt.«

\section{Dritte Metamorphose und neue Metastasen}

Im Herbst 1987 wurden die vom Strafvollzugsarchiv bislang einzeln verschickten "Musterbegründungen« zu einem »Merkheft des Strafvollzugsarchivs der Universität Bremen für Strafgefangene und Untersuchungsgefangene zusammengefaßt. Dieses >Merkheft ‘ enthielt also sämtliche im >Ratgeber abgedruckten Musterbegründungen sowie eine erst nachträglich in Bremen »im Stile des Gefangenenratgebers « hergestellte. Es wurde auf Wunsch kostenlos an Gefangene verschickt. In einzelnen Anstalten wurde es angehalten, meist aber mehr oder weniger rasch wieder freigegeben. Am I 2. I I. 1987 erließ der Leiter der Justizvollzugsanstalt Zweibrücken eine Anhalteverfügung unter ausdrücklichem Hinweis auf »den Ihnen bekannten Beschluß ... des Landgerichts Augsburg beim Amtsgericht Nördlingen Zweigstelle Donauwörth vom 20. 5. 1987«. Und am 19. Mai 1988 wies das Landgericht Zweibrücken ${ }^{26}$ meinen Antrag auf Verpflichtung der JVA Zweibrücken zur Aushändigung des Merkheftes zurück:

"Das Merkheft stellt keinen rechtlichen Ratgeber dar, der die Gefangenen sachlich über deren Rechte im Vollzug informiert. Es beruht vielmehr auf dem >Ratgeber für Gefangene`, der, wie bereits mehrfach festgestellt, das Ziel des Strafvollzuges gefährdet, 1ndem er in seiner Gesamtheıt die Absicht verfolgt, Gefangene darın zu üben, den staatlichen Strafanspruch zu unterlaufen. Die $1 \mathrm{~m}>$ Merkheft‘ gewählte Diktıon sowie die gegebene Auswahl und Darstellung von Rechtsmeinungen zielt darauf, den Gefangenen auch dann zur Gegenwehr gegen Disziplinarund Sicherungsmaßnahmen zu bestimmen, wenn ein Rechtsmittel keine hinreichende Aussıcht auf Erfolg hat...

Begriffe wie das ständig gebrauchte Wort \Knast`zeugen ebenso von der negativen Tendenz des `Merkheftes<, wie der Umstand, daß die Arbeit im Gefängnıs als Zwangsarbeıt dargestellt und der Gefangene mit keınem Wort zur Mitarbeıt am Vollzugszıel - Wiedereıngliederung nach Entlassung - aufgefordert wrrd. Beı dieser Tendenz des >Merkheftes besteht die Gefahr, daß der Besitz und das Lesen den Strafgefangenen zu einem Verhalten motiviert, das dem Vollzug zuwiderläuft.

Die Lektüre ist in ihrer Gesamtheit dazu angetan, den Gefangenen nicht zu einer sinnvollen Mitarbeit sondern lediglich zu einer destruktiven Opposition anzuhalten, ihn seme rechtswidrigen Taten vergessen und den Strafvollzug als bloße Äußerung der staatlichen Macht empfinden zu lassen «.

Damit war der bislang extremste Punkt der Verbotsjustiz erreicht ${ }^{27}$ : Nicht nur der ,Ratgeber insgesamt, sondern auch die juristischen Musterbegründungen für sich genommen waren verboten. Und zwar mit den vorher für den , Ratgeber insgesamt entwickelten Standardargumenten.

\section{Zwei Justizwunder}

In neuerer Zeit sind allerdings zwei Gerichtsentscheidungen zu verzeichnen, welche den Anfang einer differenzierteren Sichtweise darstellen könnten: Am I 8. Juli 1988

26 LG Zweibrücken, I9. 5. 1988 - I Vollz $103 / 87$.

27 Die Entscheıdung des LG Zweibrücken hatte übrıgens auch Auswırkungen auf eın Forschungsprojekt der Unıversität Bremen zum Rechtsschutz von Strafgefangenen. So schrıeb eıner der um Auskunft gebetenen Vollzugsjuristen, der Anstaltsleiter der JVA Diez, an den Fachbereich Rechtswissenschaften der Unıversität Bremen: "Ich lehne es ab, in emer Sache tätıg zu werden, für die Professor Dr. Feest verantwortlich zeschnet. Zur Begründung verwesse ich auf den anliegend übersandten Beschluß des Landgerıchts Zweibrücken vom 19.5.1988\%. Ausführlicher dazu Peter Selling, Datenschutz, Forschungsbehinderung oder was? Ein Praxisbericht aus der cmpirischen Strafvollzugsforschung, Vorgänge s/1989, S. $26-34$. 
hat die Strafvollstreckungskammer des LG Lüneburg beim Amtsgericht Celle ${ }^{28}$ die erwähnte Entscheidung der JV.A Celle aufgehoben:

"Diese Ausführungen halten rechtlicher Überprüfung nicht stand. Der Vollzugsbehörde ist zuzugeben, daß das genannte Schriftstück mit sehr klemen Druckbuchstaben erstellt worden ist und daher nur schwierıg und mit einıger Mühe gelesen werden kann. Es ist aber durchaus lesbar. Davon hat sich die Kammer selbst überzeugt. Der Inhalt dieses Schriftstücks selbst ist nicht zu beanstanden. Er erläutert dem Gefangenen seine Rechtspositıonen auf der Grundlage des Strafvollzugsgesetzes. Dies gefährdet weder bei dem Antragsteller das Ziel des Vollzuges noch die Sicherheıt oder Ordnung der Anstalt. Der angehaltene Auszug aus dem sog. Gefangenenratgeber ist daher dem Antragsteller unverzüglich auszuhändigen.«

Und am 31. August 1988 hat das OLG Zweibrücken ${ }^{29}$ folgenden Beschluß gefaßt:

"Der Letter der JVA Zweibrücken wird angewiesen, das dem Strafgefangenen T. U. G. vom Antragsteller übersandte, Merkblatt des Strafvollzugsarchıvs der Universıtät Bremen für Gefangene in Strafhaft und Untersuchungshaft mit Ausnahme des Teils >Die Rechtsmittel in Untersuchungshafe (fortlaufende Serten Nr. 40-55) auszuhändigen ... Auch bei zusammenfassender Würdigung von Inhalt, Stil und Darstellungswerse sind die aufgezeıgten vereinzelten Beanstandungen des Textes nıcht geeıgnet, eıne das Vollzugsziel gefährdende, destruktıve Oppositionshaltung berm Strafgefangenen zu erzeugen. Da es das Anliegen des >Merkheftes ist, den Gefangenen in Rechtsfällen zu beraten, in denen er sıch gegen Maßnahmen der Vollzugsbehörde wendet und deren gerıchtliche Überprüfung er verlangt, kann nıcht beanstandet werden. ... Nicht zu beanstanden ist auch die Darstellung und Auswahl der für die >Musterbegründungen angeführten Meınungen und Gerichtsentscheldungen. Vielfach ist ausdrücklich darauf verwiesen, daß die zu emer Rechtsfrage vertretenen Auffassungen streitig sind und somit der Erfolg eines Antrages bei Gericht von der dort vertretenen Meinung abhängt. $\mathrm{Da}$ in solchem Zusammenhang auch Mindermeinungen für die vorgeschlagenen Begründungstexte verwandt werden, ohne daß dies im Einzelfall kenntlich gemacht 1st, kann dem Verfasser nicht vorgeworfen werden; andernfalls würde man die Anforderungen an die Qualität eines solchen Merkheftes überspannen. Es erschennt zudem nıcht bereıts tendenziös, wenn dem sein Recht suchenden Gefangenen Entscheidungen an die Hand gegeben werden ("Arrest«, "Sicherungsmaßnahmen«), die nıcht der herrschenden Rechtsmeınung entsprechen."

Interessant ist allerdings die Begründung für das nach wie vor für notwendig gehaltene Teil-Verbot. Der auf Untersuchungsgefangene bezogene Textteil sei teilweise im Stil einer Kampfschrift gehalten, ihm liege eine erkennbar vollzugsfeindliche Auffassung zugrunde. Dafür wird (als das ausführlichste von fünf Beispielen) folgende Passage aus dem $>$ Ratgeber/Merkheft $<$ angeführt:

"Ein Tauchsieder eıgnet ssch nun mal nıcht als Ausbruchswerkzeug oder was es sonst noch an absurden Begründungen gibt. Aber vergiß nicht, Du hast es hier mit Juristen zu tun, und auch die Beschwerdeinstanz besteht aus Juristen. Und in der Denkweise von Juristen finden Logik und Vernunft kaum Platz."

Das Beispiel erscheint als Beleg dafür, daß setbst ein liberales Gericht die Grenze des Erlaubten dort zieht, wo die Rationalität von Juristen ironisiert wird.

\section{Resumé}

Was die Zulassung des ‘Ratgeber in den Gefängnissen der Bundesrepublik betrifft, ergibt sich ein differenziertes Bild: In fast allen Anstalten ist das Buch insgesamt verboten. In der JVA Bremen-Oslebshausen ist er frei zugänglich ${ }^{30}$. Im Bereich des OLG Celle ist der gesamte Rechtsmittel-Teil des >Ratgeber zugänglich. Im Bereich

28 LG Lüneburg, 18.7. 1988-17 StVK 51/88; das OLG Celle hat diese Entscheıdung bestätıgt (4.8.1988I Ws $117 / 88$ (StrVollz).

29 OLG Zweibrücken, 3r.8. 1988 - 1 Vollz (Ws) $10 / 88$.

30 Dies ist das Verdienst von Ehrhard Hoffmann, des langährigen Letters dieser Anstalt. Er gehört auch zu den Mitarbettern am Alternatıvkommentar zum Strafvollzugsgesetz. Leider ıst dieser sehr ungewöhnliche Vollzugsjurist im vorigen Jahr verstorben. 
des OLG Zweibrücken sind die Musterbegründungen für die Strafhaft, nicht jedoch für die Untersuchungshaft zugänglich. Und das OLG Stuttgart" hat entschieden, der ^Ratgeber` sei zwar insgesamt nicht zulässig und deshalb an den Absender zurückzugeben, »nach Entfernung oder veranlaßter Änderung der beanstandeten Textstellen mag dann die Schrift erneut zum Versand gebracht werden«. Unbedenklich seien »die medizinischen und rechtlichen Hinweise im zweiten Teil des Buches, auch das Kapitel Entlassung und andere Beiträge«. Schwieriger ist es wohl nach wie vor in Bayern, wiewohl auch dort zähneknirschend Konzessionen gemacht werden ${ }^{32}$. Aber auch in Nordrhein-Westfalen scheint der Konflikt noch nicht völlig ausgestanden, indem Ende 1990 in der JVA Geldern eine Broschüre der Deutschen Aidshilfe angehalten wurde, in der die Musterbegründungen des Strafvollzugsarchivs in neuester Fassung abgedruckt sind..$^{33}$

$\mathrm{Daß}$ nach wie vor großer Bedarf an juristischer Beratung von Gefangenen besteht, zeigt die Korrespondenz des Strafvollzugsarchivs. Und im Grunde ist weder der Alternativkommentar noch der $>$ Ratgeber wirklich geeignet, diesen Bedarf in seiner ganzen Breite zu befriedigen. Der Alternativkommentar wendet sich trotz aller guten Vorsätze doch in erster Linie an Juristen und ist daher (selbst unabhängig von seinem exorbitanten Preis) für die meisten Gefangenen weitgehend unbrauchbar. Und der $>$ Ratgeber ist in erster Linie für diejenigen Gefangenen brauchbar, die in der Lage sind, das rechtliche Instrumentarium im Rahmen einer umfassenderen Überlebensstrategie zu begreifen. Bei den weitaus meisten Gefangenen bringt die Versendung von Musterbegründungen die Gefahr, daß sie sich Illusionen über den gerichtlichen Rechtsschutz machen. ${ }^{34}$ Deshalb wären andere, angemessenere Rechtsratgeber für Gefangene sehr erwünscht. ${ }^{35}$

Unabhängig von diesen Überlegungen bleibt jedoch festzuhalten, daß die Verbotsgeschichte des >Ratgebers^ kein Ruhmesblatt für die deutsche Justiz darstellt. Zwei Traditionen treffen hier zusammen und verstärken sich in unheilvoller Weise: Zum einen die faktisch immer noch anzutreffende Tendenz, den Strafvollzug als besonderes Gewaltverhältnis anzusehen und die Grundrechte von Gefangenen in genauerer Prüfung nicht standhaltenden Weise zu beschränken. Zum anderen die spezifischere Verbotstradition einer $\ddot{A r}$, in der hinter jeder »staatsfeindlichen « Äußerung das Gespenst des Terrorismus und seiner Sympathisanten auftauchte. Dieser Aufsatz sollte einen kleinen Beitrag zur Dokumentation und Kritik dieser Tendenzen leisten.

31 OLG Stuttgart, 5.4. $1988-5$ W/s $17 / 88$.

32 Auf meine Beschwerde im eingangs erwähnten Fall erhielt ıch am 29.9.1989 folgenden Brief des Anstaltsletters der JVA Bernau: "Ich teile zwar nıcht Ihre Meınung, daß die Aushändigung der von Ihrem Mitarbeıter übersandten Merkblätter an Strafgefangene unbedenklich wäre und vermag diese Ansıcht in dieser Allgemeinheit auch nicht der von Ihnen zitierten Rechtsprechung zu entnehmen. Gleıchwohl darf ıch Ihnen mıtteilen, daß Herrn D. die Merkblätter unter Zurückstellung der hıer nach wie vor bestehenden Bedenken zwıschenzeitlich ausgehändigt wurden".

33 Deutsche AIDS-Hilfe e.V., Positıv, was nun? Ein Ratgeber für Menschen mıt HIV/AIDS in Haft, Berlin 1990. Die Anstaltsentscheidung ist inzwischen vom LG Kleve aufgehoben worden (21.3.1991 - 2 Vollz 4/91).

34 Dies ist auch eın Resultat der von Peter Selling und mır durchgefübrten Untersuchung zum gerıchtlichen Rechtsschutz von Gefangenen (erscheınt dernnächst).

35 Vorbilder könnten sem: das von Gerard de Jonge und Rino Verpalen verfaßte umfangreiche Handboek voor gedeuneerden en ter beschikıng gestelden. Den Haag 1982, aber auch das noch allgemeınverständlichere Büchleın "How to survive in the nick « des britsschen Ex-Gefangenen John Marshall (London 1974). 